\title{
CARACTERIZAÇÃO DA PARTICIPAÇÃO FEMININA NO MERCADO DE TRABALHO: UMA ANÁLISE DE DECOMPOSIÇÃO*
}

\author{
Luiz Guilherme Scorzafave ${ }^{\S}$ \\ Naércio Menezes-Filho
}

\begin{abstract}
RESUMO
O objetivo deste trabalho é realizar uma decomposição da evolução da participação feminina no mercado de trabalho brasileiro. Particularmente, queremos saber a importância da alteração da incidência da TPFT dentro de subgrupos da população feminina, bem como a contribuição da mudança do tamanho de tais grupos no crescimento da participação feminina. Entre os principais resultados, destacamos que a alteração da incidência da participação nos diferentes subgrupos respondeu pela maior parte do crescimento da TPFT no período de 1982 a 2002. Por outro lado, o aumento no tamanho dos grupos com mulheres mais educadas contribuiu com o restante da evolução.
\end{abstract}

Palavras-chave: decomposição, mulher, participação.

\begin{abstract}
The objective of this paper is to decompose the evolution of the female's labor force participation rate (LFPR) in the Brazilian labor market. Particularly, we want to investigate the importance of changes in LFPR within female sub-groups, as well as to know if size changes of such groups contribute to explain the growth of LFPR between women. Among the main results, we find that changes in the LFPR within sub-groups are responsible for more than a half of the LFPR variation between 1982 and 2002. On the other hand, the increases in the size of groups containing more educated women have contributed with the remainder.
\end{abstract}

Key-words: decomposition, woman, participation.

JEL classification: J82, J21, J16.

\footnotetext{
* Os autores agradecem a Rafael Satake pela excelente assistência de pesquisa.

$\S$ Professor da FEA-RP/USP. Endereço para contato: Av. dos Bandeirantes, 3900 - FEA - bloco C - sala 65 - Ribeirão Preto - SP. CEP 14040-900.E-mail: scorza@usp.br.

¿ Professor do IBMEC-SP e da FEA-USP. E-mail: naercioamf@isp.edu.br.
}

Recebido em setembro de 2004. Aceito em setembro de 2005. 


\section{INTRODUÇÃO}

Na segunda metade do século XX, diversos países experimentaram um significativo crescimento da participação feminina no mercado de trabalho. Killingsworth e Heckman (1986) apontam que a taxa de participação feminina na força de trabalho (TPFT) nos Estados Unidos passou de $60 \%$ para $71 \%$ entre 1980 e 1995 . No Canadá, entre 1970 e 1980, a TPFT passou de $40 \%$ para $52 \%$. Este processo também vem ocorrendo no Brasil. Por exemplo, Scorzafave e Menezes-Filho (2001) mostram que a TPFT feminina passou de 41\% para 54\% entre 1982 e 1997, ou seja, um crescimento de cerca de 13 pontos porcentuais. Os mesmos autores descrevem a evolução da taxa de participação desagregada por diversos indicadores como região de residência, escolaridade, idade, entre outros.

A rapidez deste processo de inserção feminina no mercado de trabalho requer um olhar mais atento sobre este grupo. Assim, de modo geral, o objetivo deste artigo é conhecer mais detalhadamente as características das mulheres que participam do mercado de trabalho, incorporando novos aspectos ainda não abordados no caso brasileiro. Adicionalmente, para levar em conta o crescimento da participação ao longo do tempo, realiza-se esta análise em períodos distintos de tempo, a fim de construir um quadro mais nítido de como o perfil da mulher no mercado de trabalho vem se alterando.

Alguns trabalhos já procuraram caracterizar a atividade feminina no caso brasileiro. Por exemplo, Wajnman, Queiroz e Liberato (1998) mostram o crescimento da atividade feminina e tentam caracterizar de que modo se deu essa inserção nos anos noventa no Brasil. Concluem que o maior crescimento se deu para as mulheres trabalhando por conta própria no comércio de mercadorias (comércio ambulante de cosméticos e alimentos), domésticas na prestação de serviços, funcionárias públicas das atividades sociais (professoras, médicas, enfermeiras, serventes, faxineiras e cozinheiras) e na administração pública (ajudante, auxiliar administrativa, administradoras). Além disso, ressaltam que a categoria das conta própria no comércio de mercadorias foi responsável por absorver mulheres inativas e desempregadas. Assim, de modo geral, o setor de comércio/serviços parece ter sido o maior responsável pela geração de empregos.

Bruschini e Lombardi (1996) descrevem a ocupação feminina sob diversos aspectos, analisando inclusive a distribuição das mulheres trabalhadoras segundo faixas de rendimento e horas de trabalho semanais, além de realizar desagregações por setores de atividade e grupos de ocupação. Entre os principais resultados, elas identificam no crescimento do setor terciário uma gama maior de possibilidades de inserção das mulheres no mercado de trabalho (comércio, serviços, bancos), compensando os efeitos negativos gerados pela crise dos anos 80. Esse crescimento ocorreu tanto no setor informal da economia (comércio ambulante, representantes de venda) quanto no setor formal, em atividades ligadas ao setor público.

Este artigo procura abordar outros aspectos da discussão. Assim, iremos nos concentrar na Taxa de Participação na Força de Trabalho e, ao contrário dos textos citados, vamos nos preocupar menos com o destino das mulheres que decidiram entrar no mercado de trabalho e mais com os subgrupos que têm contribuído para o aumento da participação feminina. Para tanto, dividiremos as mulheres em diversas categorias e iremos comparar a evolução do tamanho de cada uma das categorias e a evolução da TPFT das mesmas em dois anos distintos, 1982 e 2002. Assim, poderemos saber o quanto da variação ocorrida na TPFT feminina neste intervalo de tempo pode ser explicada pela mudança do tamanho dos grupos e pela alteração da participação dentro dos grupos.

Cabe ressaltar que apesar de abordar o mesmo tema, no caso a evolução da TPFT, o presente trabalho utiliza uma metodologia diferente da empregada em Scorzafave e Menezes-Filho (2001). Eles utilizam um método paramétrico para decompor a evolução da TPFT entre 1982 e 1997, con- 
cluindo que metade da evolução se devia à alteração das características que afetavam a participação, enquanto a outra metade correspondia à alteração nos coeficientes da equação de participação. Por sua vez, o presente artigo procura decompor a evolução da participação feminina implementando duas metodologias alternativas (não-paramétricas) que permitem avaliar em que medida a alteração do tamanho relativo dos grupos socioeconômicos na população afetou a evolução da TPFT e quais grupos de mulheres experimentaram o maior crescimento da TPFT no período.

Por fim, cabe destacar que, até onde é de nosso conhecimento, este é o primeiro artigo a aplicar as metodologias discutidas nas seções 2 e 3 para analisar a evolução da participação feminina no mercado de trabalho.

\section{DECOMPOSIÇÃo POR VARIÁVEIS}

Nossa base de dados é a Pesquisa Nacional por Amostra de Domicílios (PNAD), e a amostra é constituída por mulheres entre 25 e 64 anos de idade nos anos de 1982 e 2002. O conceito de TPFT que utilizamos incorpora tanto empregados como desempregados como proporção da população em idade ativa. Também cabe destacar que a PNAD sofreu mudança de metodologia a partir de 1992, redefinindo os conceitos de atividade econômica. Assim, realizamos os ajustes necessários na PNAD de 2002 para reconstituir o conceito de participação usado até a PNAD de 1990.

Especificamente, consideramos como não ocupados, mas economicamente ativos:

a) os trabalhadores na produção para o próprio consumo que tinham tomado providência para conseguir trabalho na semana de 22 a 28/09/2002;

b) os trabalhadores na construção para uso próprio que tinham tomado providência para conseguir trabalho na semana de 22 a 28/09/2002;

c) os trabalhadores não remunerados (no trabalho principal) que tinham tomado alguma providência para conseguir trabalho na semana de 22 a 28/09/2002.

Por outro lado, consideramos como ocupados:

a) os trabalhadores não remunerados (no trabalho principal) que trabalhavam mais de 14 horas por semana;

b) os trabalhadores não remunerados (no trabalho principal) que apesar de trabalharem entre $1 \mathrm{e}$ 14 horas semanais, tinham mais de um emprego e a renda deste emprego secundário era positiva;

c) os trabalhadores não remunerados (no trabalho principal) que trabalhavam entre 1 a 14 horas no trabalho principal e tinham outro emprego em que trabalhavam mais de 15 horas por semana. ${ }^{1}$

Feitas estas considerações metodológicas, voltemos à nossa discussão. Como já dissemos, queremos saber como os diferentes grupos socioeconômicos de mulheres contribuíram para a evolução da TPFT feminina entre 1982 e 2002. Para tanto, podemos realizar uma análise de decomposição da variação da TPFT feminina em dois componentes: (a) qual a parcela da variação da TPFT que se deve à mudança no tamanho de cada grupo socioeconômico?; (b) qual a parcela da mudança da TPFT que se deve à alteração da TPFT dentro de cada grupo socioeconômico? Para exemplificar, tomemos o caso de separação das mulheres em dois subgrupos: residentes em áreas urbanas e rurais. Inicialmente, vamos verificar a TPFT das mulheres de cada subgrupo em 1982 e 2002

1 Para mais detalhes, ver Guerra (1997). 
$\left(\mathrm{TPFT}_{\mathrm{U}}{ }^{82}, \mathrm{TPFT}_{\mathrm{R}}{ }^{82}, \mathrm{TPFT}_{\mathrm{U}}{ }^{02}, \mathrm{TPFT}_{\mathrm{R}}{ }^{02}\right)$, bem como o tamanho relativo de cada subgrupo relativamente ao total de mulheres $\left(\mathrm{POP}_{\mathrm{U}}^{82}, \mathrm{POP}_{\mathrm{R}}^{82}, \mathrm{POP}_{\mathrm{U}}^{02}, \mathrm{POP}_{\mathrm{R}}^{02}\right)$.

TPFT $_{\mathrm{U}}{ }^{82}$ é a TPFT entre as mulheres residentes em áreas urbanas em 1982; $\mathrm{POP}_{\mathrm{U}}{ }^{82}$ é a proporção de mulheres em áreas urbanas em relação ao total de mulheres em 1982. Analogamente, definimos $\mathrm{TPFT}_{\mathrm{R}}{ }^{82}$ e $\mathrm{POP}_{\mathrm{R}}^{82}$ para as áreas rurais, bem como os mesmos conceitos para 2002. Deste modo, podemos escrever:

$$
\begin{gathered}
\mathrm{TPFT}^{82}=\mathrm{TPFT}_{\mathrm{U}}{ }^{82} * \mathrm{POP}_{\mathrm{U}}{ }^{82}+\mathrm{TPFT}_{\mathrm{R}}{ }^{82} * \mathrm{POP}_{\mathrm{R}}{ }^{82} \\
\mathrm{TPFT}^{02}=\mathrm{TPFT}_{\mathrm{U}}{ }^{02} * \mathrm{POP}_{\mathrm{U}}{ }^{02}+\mathrm{TPFT}_{\mathrm{R}}{ }^{02} * \mathrm{POP}_{\mathrm{R}}{ }^{02}
\end{gathered}
$$

A variação da TPFT entre 1982 e 2002 pode ser definida como:

$$
\begin{aligned}
& \Delta \mathrm{TPFT}=\mathrm{TPFT}^{02}-\mathrm{TPFT}^{82}= \\
& =\Sigma_{\mathrm{i}}\left(\mathrm{TPFT}_{\mathrm{i}}^{02}-\mathrm{TPFT}_{\mathrm{i}}^{82}\right) * \mathrm{POP}_{\mathrm{i}}^{82}+\Sigma_{\mathrm{i}}\left(\mathrm{POP}_{\mathrm{i}}^{02}-\mathrm{POP}_{\mathrm{i}}^{82}\right) * \mathrm{TPFT}_{\mathrm{i}}^{02} \\
& =\text { Efeito Incidência } \quad+\quad \text { Efeito Tamanho } \\
& =\text { E.I. }+ \text { E.T. }
\end{aligned}
$$

onde $\mathrm{i}=\mathrm{U}, \mathrm{R}$.

O primeiro termo da equação acima indica qual teria sido a mudança na TPFT para o conjunto das mulheres entre 1982 e 2002 se a única alteração ocorrida no período fosse a incidência da TPFT dentro de cada grupo (urbano e rural), assumindo que a distribuição das mulheres entre áreas urbanas e rurais não tivesse se alterado. O segundo termo avalia a parcela da mudança da TPFT decorrente da alteração no tamanho relativo dos grupos entre 1982 e 2002, avaliada às taxas de 2002.

A seguir, apresentamos a aplicação da decomposição para diferentes recortes da amostra.

\subsection{Urbana/rural e metropolitana}

Na Tabela 1 mostramos os resultados da separação das mulheres de acordo com dois critérios: residentes em áreas rurais e urbanas e residentes ou não em regiões metropolitanas. A primeira e a terceira coluna da tabela apontam a proporção de mulheres em cada grupo; a segunda e a quarta indicam a TPFT de cada grupo nos dois anos. A quinta e sexta colunas mostram o efeito tamanho e o efeito incidência, respectivamente. A sétima e a oitava colunas indicam os mesmos efeitos como proporção da variação da TPFT das mulheres como um todo no período. ${ }^{2}$ A última coluna apresenta a soma da sétima e da oitava colunas e fornece a contribuição de cada grupo para a evolução da TPFT no período.

O primeiro aspecto a ser considerado é que a TPFT das mulheres aumentou de 0,408 em 1982 para 0,588 em 2002, ou seja, um crescimento de 18 pontos porcentuais, que indica a continuidade do crescimento da TPFT até 1997, apontado por Scorzafave e Menezes-Filho (2001).

Por sua vez, considerando a divisão das mulheres das áreas urbana e rural, houve um aumento significativo na proporção que residia em regiões urbanas. Com relação à TPFT, ela é maior nas áreas urbanas do que nas rurais nos dois períodos. Notamos, adicionalmente, que o crescimento

2 As discrepâncias entre os valores de $\mathrm{TPFT}^{02}-\mathrm{TPFT}^{82}$ devem-se ao arredondamento dos valores de TPFT e \% Pop. 
porcentual da TPFT foi praticamente o mesmo nos dois grupos (cerca de 41\%). Por outro lado, a continuidade do processo de urbanização elevou a proporção de mulheres nas regiões urbanas. Desta forma, a maior parte da variação da TPFT no período $(94,1 \%)$ pode ser atribuída ao efeito incidência. Além disso, a evolução da TPFT relativa às áreas urbanas correspondeu a 111,8\% da evolução da TPFT observada para todas as mulheres.

Tabela 1 - TPFT e tamanho dos grupos por região de residência - 1982-2002

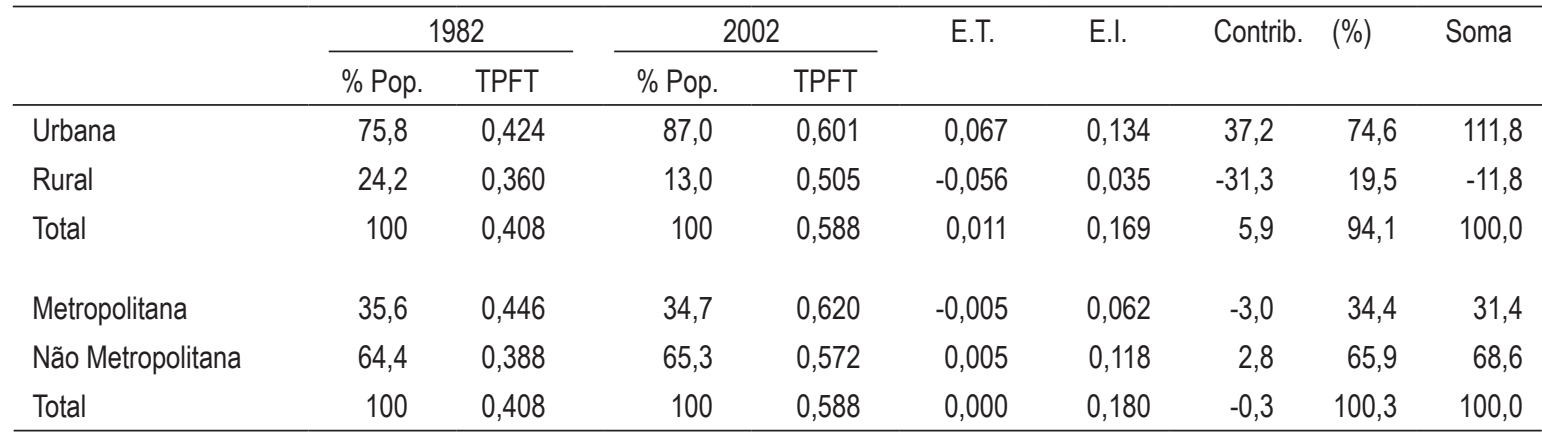

E.T: efeito tamanho.

E.I.: efeito incidência.

Em relação às áreas metropolitanas, há uma estabilidade na proporção de mulheres, o que faz com que uma parcela muito pequena da variação da TPFT esteja relacionada ao efeito tamanho. Por outro lado, a maior proporção de mulheres em áreas não metropolitanas associada ao crescimento da TPFT deste grupo faz com que este grupo responda por 65,9\% da variação da TPFT no período. Agregando os dois efeitos, percebemos que a evolução dos tamanhos relativos dos grupos e da incidência da TPFT em cada grupo faz com que as áreas não metropolitanas contribuam com 68,6\% do diferencial de TPFT no período.

\subsection{Posição na família}

Em seguida, desagregamos os dados de acordo com a estrutura da família. Ou seja, verificamos o que ocorreu com a composição do grupo das mulheres chefes, cônjuges e em outras posições na família, bem como o que ocorreu com a TPFT em cada um destes subgrupos. ${ }^{3}$ Por fim, calculamos a parcela do aumento da TPFT atribuível aos efeitos tamanho e incidência, bem como qual dos grupos responde pela maior parte da evolução da TPFT no período.

$\mathrm{Na}$ Tabela 2, percebemos que o grupo que mais cresceu foi o das chefes de família, e o que mais diminuiu foi o das mulheres cônjuges. Apesar disso, a proporção de mulheres cônjuges continuou muito elevada em relação ao total de mulheres. Os outros grupos não tiveram grandes alterações no seu tamanho relativo.

3 Nesse caso, a análise inclui somente mulheres chefe, cônjuges, filhas e com outro grau de parentesco com o chefe da família. 
Tabela 2 - TPFT e tamanho dos grupos por posição na família - 1982-2002

\begin{tabular}{|c|c|c|c|c|c|c|c|c|c|}
\hline \multirow[b]{3}{*}{ Chefe } & \multicolumn{2}{|c|}{1982} & \multicolumn{2}{|c|}{2002} & \multirow{3}{*}{$\begin{array}{c}\text { E.T. } \\
0,068\end{array}$} & \multirow{3}{*}{$\begin{array}{l}\text { E.I. } \\
0,011\end{array}$} & \multicolumn{2}{|c|}{ Contrib. (\%) } & \multirow[t]{2}{*}{ Soma } \\
\hline & \% Pop. & TPFT & \% Pop. & TPFT & & & & & \\
\hline & 14,7 & 0,601 & 24,8 & 0,678 & & & 36,8 & 6,1 & 43,0 \\
\hline Cônjuge & 74,1 & 0,333 & 64,5 & 0,536 & $-0,052$ & 0,151 & $-27,9$ & 81,4 & 53,5 \\
\hline Filha & 7,2 & 0,695 & 7,8 & 0,729 & 0,005 & 0,002 & 2,5 & 1,3 & 3,8 \\
\hline Outro Parente & 4,0 & 0,407 & 29 & 0,537 & $-0,006$ & 0,005 & $-3,1$ & 2,8 & $-0,3$ \\
\hline Total & 100 & 0,402 & 100 & 0,586 & 0,015 & 0,170 & 8,4 & 91,6 & 100,0 \\
\hline
\end{tabular}

No caso da TPFT, o maior aumento se deu entre as cônjuges e o menor entre as filhas. De modo geral, o efeito incidência é responsável por mais de $90 \%$ da variação da TPFT no período; ou seja, a alteração no tamanho relativo dos grupos responde apenas por reduzida parcela do diferencial de TPFT entre os anos. Por outro lado, a ainda alta proporção de mulheres cônjuges em 2002 e o forte crescimento da TPFT entre as mesmas fazem com que respondam por cerca de 53,5\% do aumento da TPFT no período. Já o crescimento da proporção de chefes responde pela maior parte dos 43,0\% de aumento de TPFT atribuível a este grupo.

\subsection{Número de filhos}

A seguir, investigamos o número de filhos presentes no domicílio entre as mulheres, fossem elas chefes ou cônjuges. A Tabela 3 ilustra os resultados. A grande mudança no período foi a redução da proporção de mulheres com quatro ou mais filhos no domicílio (passaram de 29,0\% para $8,9 \%)$ e o crescimento na fração de mulheres com um ou dois filhos residentes no mesmo domicílio (de $40,2 \%$ para $58,6 \%$ ).

No tocante à participação, quanto maior o número de filhos, menos as mulheres participam do mercado de trabalho, tanto em 1982 quanto em 2002. Vale notar, entretanto, que as mulheres com muitos filhos tiveram aumento da TPFT de menor magnitude que as demais mulheres com filhos. Da mesma forma que para a análise por posição na família, no caso da divisão das mulheres por número de filhos, o efeito incidência responde por mais de $88 \%$ da variação total da TPFT, sendo que a maior contribuição para o efeito incidência veio das mulheres com 2 filhos.

Tabela 3 - TPFT e tamanho dos grupos por número de filhos - chefes e cônjuges 1982-2002

\begin{tabular}{|c|c|c|c|c|c|c|c|c|c|}
\hline & \multicolumn{2}{|c|}{1982} & \multicolumn{2}{|c|}{2002} & \multirow[t]{2}{*}{ E.T. } & \multirow[t]{2}{*}{ E.I. } & \multirow[t]{2}{*}{ Contrib. } & \multirow[t]{2}{*}{$(\%)$} & \multirow[t]{2}{*}{ Soma } \\
\hline & \% Pop. & TPFT & \% Pop. & TPFT & & & & & \\
\hline 0 & 13,9 & 0,446 & 17,9 & 0,567 & 0,023 & 0,017 & 11,4 & 8,5 & 19,9 \\
\hline 1 & 18,4 & 0,403 & 28,8 & 0,596 & 0,062 & 0,035 & 31,1 & 17,9 & 49,0 \\
\hline 2 & 21,8 & 0,372 & 29,8 & 0,593 & 0,048 & 0,048 & 24,0 & 24,3 & 48,2 \\
\hline 3 & 16,7 & 0,358 & 14,6 & 0,559 & $-0,012$ & 0,034 & $-5,9$ & 16,9 & 11,0 \\
\hline 4 & 10,8 & 0,353 & 5,2 & 0,500 & $-0,028$ & 0,016 & $-14,0$ & 8,0 & $-6,0$ \\
\hline 5 ou mais & 18,2 & 0,339 & 3,7 & 0,484 & $-0,070$ & 0,026 & $-35,4$ & 13,3 & $-22,1$ \\
\hline Total & 100 & 0,378 & 100 & 0,576 & 0,022 & 0,177 & 11,2 & 88,8 & 100,0 \\
\hline
\end{tabular}


Por outro lado, a queda drástica do número de mulheres com 5 ou mais filhos mais do que compensa o aumento da TPFT deste grupo, fazendo com que seja o grupo que mais perde representatividade entre as mulheres participantes do mercado de trabalho. É importante destacar que a evolução da TPFT neste caso foi de 19,9 pontos porcentuais, pois estamos considerando nesta tabela apenas a TPFT conjunta de chefes e cônjuges.

Entretanto, como os resultados da Tabela 3 tratam conjuntamente chefes e cônjuges, isto pode mascarar algumas tendências diferenciadas destes dois grupos. Por exemplo, esperamos que as chefes sem filhos participem mais do que as cônjuges sem filhos. Desta forma, a Tabela 4 ilustra os resultados desagregados entre mulheres chefes e cônjuges.

Desagregando os dados, percebemos que mais de 50\% das chefes têm até um filho em 1982 e 2002. Entretanto, em 2002 cai a fração de chefes sem filhos e a de chefes com 4 ou mais filhos. Por outro lado, cresce o número de chefes com 1 e 2 filhos. Este processo parece estar ligado à entrada das novas gerações na nossa amostra. No caso das chefes com 1 filho, cerca de $31 \%$ possuíam mais de 55 anos em 1982 e 25\% tinham entre 25 e 34 anos. Em 2002, estes números eram, respectivamente, $22 \%$ e $27 \%$. No caso das cônjuges, é novamente o grupo das mulheres com 1 ou 2 filhos que apresenta maior crescimento. Vale notar a impressionante redução na proporção de mulheres com 5 ou mais filhos (queda de $20 \%$ para $4 \%$ entre 1982 e 2002).

Tabela 4 - TPFT e tamanho dos grupos por número de filhos - 1982-2002

\begin{tabular}{|c|c|c|c|c|c|c|c|c|c|}
\hline \multirow[b]{2}{*}{ Chefes } & \multicolumn{2}{|c|}{1982} & \multicolumn{2}{|c|}{2002} & \multirow[t]{2}{*}{ E.T. } & \multirow[t]{2}{*}{ E.I. } & \multirow[t]{2}{*}{ Contrib. } & \multirow[t]{2}{*}{$(\%)$} & \multirow[t]{2}{*}{ Soma } \\
\hline & \% Pop. & TPFT & $\%$ Pop. & TPFT & & & & & \\
\hline 0 & 24,7 & 0,631 & 22,5 & 0,655 & $-0,014$ & 0,006 & $-18,3$ & 7,7 & $-10,6$ \\
\hline 1 & 27,0 & 0,578 & 35,7 & 0,680 & 0,059 & 0,028 & 75,6 & 35,3 & 110,9 \\
\hline 2 & 18,6 & 0,602 & 24,3 & 0,707 & 0,040 & 0,020 & 51,3 & 25,1 & 76,4 \\
\hline 3 & 11,9 & 0,59 & 11,0 & 0,678 & $-0,006$ & 0,010 & $-8,1$ & 13,4 & 5,3 \\
\hline 4 & 8,1 & 0,633 & 4,0 & 0,647 & $-0,026$ & 0,001 & $-33,7$ & 1,5 & $-32,2$ \\
\hline 5 ou mais & 9,6 & 0,572 & 2,5 & 0,635 & $-0,045$ & 0,006 & $-57,5$ & 7,7 & $-49,8$ \\
\hline Total & 100 & 0,601 & 100 & 0,678 & 0,007 & 0,071 & 9,4 & 90,6 & 100,0 \\
\hline \multicolumn{10}{|l|}{ Cônjuges } \\
\hline 0 & 11,8 & 0,369 & 16,1 & 0,520 & 0,023 & 0,018 & 11,1 & 8,8 & 19,9 \\
\hline 1 & 16,7 & 0,347 & 26,1 & 0,552 & 0,052 & 0,034 & 25,7 & 16,9 & 42,6 \\
\hline 2 & 22,5 & 0,335 & 32,0 & 0,560 & 0,053 & 0,051 & 26,1 & 25,0 & 51,1 \\
\hline 3 & 17,7 & 0,327 & 16,0 & 0,528 & $-0,009$ & 0,036 & $-4,5$ & 17,6 & 13,1 \\
\hline 4 & 11,4 & 0,314 & 5,7 & 0,460 & $-0,026$ & 0,017 & $-13,0$ & 8,2 & $-4,7$ \\
\hline 5 ou mais & 19,9 & 0,316 & 4,1 & 0,448 & $-0,071$ & 0,026 & $-35,0$ & 13,0 & $-22,0$ \\
\hline Total & 100 & 0,333 & 100 & 0,536 & 0,021 & 0,181 & 10,5 & 89,5 & 100,0 \\
\hline
\end{tabular}

No que diz respeito à TPFT, notamos a discrepância dos valores entre chefes e cônjuges para todos os números de filhos. No caso das cônjuges, parece haver uma relação inversa entre o número de filhos e a TPFT, principalmente em 1982. Entretanto, tal relação não fica evidente para as chefes. Porém, quando comparamos a evolução da TPFT percebemos que tanto para chefes como para cônjuges a variação absoluta foi maior para as com até três filhos.

O efeito incidência corresponde a cerca de $90 \%$ da variação da TPFT. Entretanto, os resultados de cada uma das categorias de número de filhos são bem diferentes. Por exemplo, o efeito tamanho para as chefes com 1 filho responde por cerca de 91,7\% do diferencial, o que, agregado com 
a contribuição referente ao efeito tamanho, faz com que este grupo tenha respondido por mais de $100 \%$ da variação da TPFT. $^{4}$ Além disso, as chefes com 2 e as com 3 filhos também contribuíram positivamente para o aumento da TPFT do grupo, enquanto que as mulheres com 4 ou mais filhos foram o destaque negativo, devido ao efeito tamanho negativo.

Já entre as cônjuges, aquelas com 2 filhos foram as principais responsáveis pela elevação da TPFT. Cabe destacar aqui a diferença entre chefes e cônjuges sem filhos. Enquanto para as primeiras o tamanho relativo do grupo contribuiu para que elas perdessem espaço entre as mulheres participantes do mercado de trabalho, para as últimas, tanto o efeito tamanho quanto o efeito incidência foram positivos. Por fim, vale notar que as cônjuges com 4 ou mais filhos contribuíram negativamente para a evolução da TPFT, devido à sua redução numérica captada pelo efeito tamanho.

\subsection{Idade}

Agora, analisaremos como o grupo das mulheres é dividido conforme a idade e calcularemos a TPFT nas diversas faixas etárias.

Tabela 5 - TPFT e tamanho dos grupos por idade - 1982-2002

\begin{tabular}{|c|c|c|c|c|c|c|c|c|c|}
\hline & \multicolumn{2}{|c|}{1982} & \multicolumn{2}{|c|}{2002} & \multirow[t]{2}{*}{ E.T. } & \multirow[t]{2}{*}{ E.I. } & \multirow[t]{2}{*}{ Contrib. } & \multirow[t]{2}{*}{$(\%)$} & \multirow[t]{2}{*}{ Soma } \\
\hline & \% Pop. & TPFT & \% Pop. & TPFT & & & & & \\
\hline $25-29$ & 21,1 & 0,467 & 17,3 & 0,654 & $-0,025$ & 0,039 & $-13,9$ & 21,9 & 8,0 \\
\hline $30-34$ & 17,6 & 0,463 & 16,1 & 0,670 & $-0,010$ & 0,036 & $-5,5$ & 20,2 & 14,7 \\
\hline $35-39$ & 14,7 & 0,466 & 15,9 & 0,673 & 0,008 & 0,030 & 4,4 & 16,9 & 21,2 \\
\hline $40-44$ & 12,8 & 0,439 & 14,5 & 0,655 & 0,011 & 0,028 & 6,0 & 15,4 & 21,4 \\
\hline $45-49$ & 10,9 & 0,389 & 12,2 & 0,599 & 0,008 & 0,023 & 4,2 & 12,7 & 16,9 \\
\hline $50-54$ & 9,5 & 0,325 & 10,0 & 0,492 & 0,003 & 0,016 & 1,5 & 8,8 & 10,3 \\
\hline $55-59$ & 7,5 & 0,267 & 7,7 & 0,374 & 0,001 & 0,008 & 0,3 & 4,5 & 4,8 \\
\hline $60-64$ & 5,8 & 0,177 & 6,4 & 0,239 & 0,001 & 0,004 & 0,7 & 2,0 & 2,8 \\
\hline Total & 100 & 0,408 & 100 & 0,588 & $-0,004$ & 0,184 & $-2,4$ & 102,4 & 100,0 \\
\hline Idade Média & 40,0 & & 40,8 & & & & & & \\
\hline
\end{tabular}

Primeiramente, percebemos que houve queda da participação relativa das mulheres jovens, sendo que as mulheres entre 35 e 49 anos ganharam espaço. Desta forma, o processo de envelhecimento da população brasileira está em parte refletido em nossa amostra, cuja idade média aumentou 0,8 anos no período. Com relação à TPFT, todas as faixas etárias apresentaram crescimento. Entretanto, este foi mais forte entre as mulheres de 30 a 49 anos e menor entre as mais idosas. $\mathrm{Na}$ divisão por idade, o efeito incidência é responsável por toda a variação da TPFT, dado que as mudanças de tamanho relativo dos grupos são pequenas, por estarem ligadas a aspectos demográficos. Combinando o efeito tamanho e incidência, as mulheres de 40 a 44 anos foram as que mais contribuíram para a evolução da TPFT.

4 Nesse caso, a parcela de cada grupo foi calculada separadamente para chefes e cônjuges. O aumento observado de TPFT apenas entre as chefes no período foi de 7,8 pontos porcentuais e, entre as cônjuges, de 20,3 pontos porcentuais. 


\subsection{Cor da pele}

A seguir, desagregamos os resultados de acordo com a cor da pele. Em termos de tamanho dos grupos, a única mudança entre 1982 e 2002 é uma pequena redução na proporção de mulheres de cor preta, branca e amarela e um aumento nas de cor parda. Mesmo assim, em 2002, a maior parte das mulheres era de cor branca. ${ }^{5}$ Deste modo, novamente se justifica o pequeno efeito tamanho encontrado neste caso.

Tabela 6 - TPFT e tamanho dos grupos por cor da pele - 1982-2002

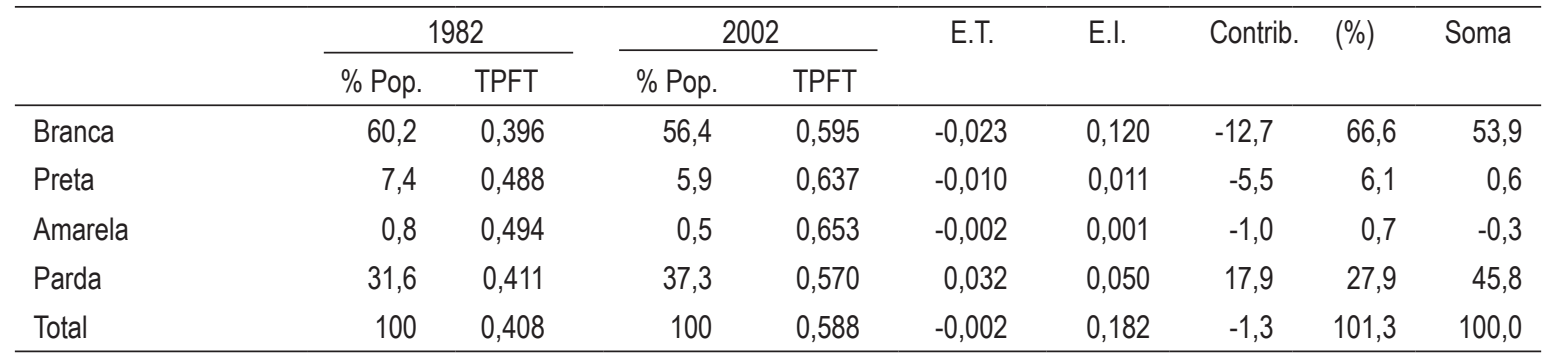

Por sua vez, a TPFT difere pouco entre os grupos, principalmente em 2002, pois o grupo com participação mais baixa em 1982 - as mulheres brancas - foi o que obteve maior crescimento da TPFT. Já as que possuíam maior TPFT em 1982 - mulheres amarelas - tiveram o menor crescimento da TPFT. Em termos do efeito incidência, as maiores contribuições vêm de mulheres brancas e pardas, dado que as mesmas eram a maioria em 2002 e experimentaram uma elevação considerável da TPFT no período.

\subsection{Escolaridade}

Finalmente, desagregamos os resultados por nível educacional das mulheres. O primeiro aspecto a ressaltar é que a escolaridade média da amostra aumentou cerca de 2,7 anos no período. Ao desagregar por faixas educacionais, percebemos uma forte redução da proporção de mulheres menos educadas, sendo que o grupo que mais cresceu foi o das mulheres com 8 a 11 anos de estudo, seguido pelo grupo daquelas com 12 ou mais anos de estudo, que dobraram sua participação relativa. Com relação à TPFT, há uma relação direta entre escolaridade e participação no mercado de trabalho. Comparando a evolução dos grupos, percebemos que as mulheres com 4 a 7 anos de estudo foram as que mais aumentaram sua TPFT e as que menos aumentaram foram as mais educadas.

No que diz respeito ao efeito tamanho, o resultado da desagregação por grupos de educação se mostra distinto do encontrado para os outros cortes da amostra, pois cerca de $38 \%$ do diferencial da TPFT é explicado por mudança no tamanho dos grupos. O aumento na proporção de mulheres com mais de 8 anos de estudo, dada a elevada TPFT das mesmas, é responsável por este resultado. Assim, aumentou o tamanho dos grupos que participavam mais ativamente do mercado de trabalho em detrimento daqueles que tinham baixa inserção. Por outro lado, os dois grupos com menor educação contribuem com a maior parte do efeito incidência, dado que experimentaram vigoroso crescimento da TPFT no período. Entretanto, quando combinamos os dois efeitos, os resultados

5 Na realidade, tais diferenças, provavelmente, estão relacionadas ao processo de coleta das informações, já que na PNAD é o próprio indivíduo que reporta a sua cor de pele. 
mudam, sendo que as maiores contribuições para o aumento da TPFT vêm das mulheres com 8 a 11 anos de estudo.

Tabela 7 - TPFT e tamanho dos grupos por anos de estudo - 1982-2002

\begin{tabular}{|c|c|c|c|c|c|c|c|c|c|}
\hline & \multicolumn{2}{|c|}{1982} & \multicolumn{2}{|c|}{2002} & \multirow[t]{2}{*}{ E.T. } & \multirow[t]{2}{*}{ E.I. } & \multirow[t]{2}{*}{ Contrib. } & \multirow[t]{2}{*}{$(\%)$} & \multirow[t]{2}{*}{ Soma } \\
\hline & \% Pop. & TPFT & $\%$ Pop. & TPFT & & & & & \\
\hline 0 a 3 & 51,1 & 0,342 & 26,3 & 0,431 & $-0,107$ & 0,045 & $-59,3$ & 25,1 & $-34,2$ \\
\hline 4 a 7 & 29,1 & 0,388 & 29,4 & 0,543 & 0,002 & 0,045 & 0,9 & 25,1 & 26,0 \\
\hline 8 a 11 & 14,3 & 0,534 & 32,7 & 0,670 & 0,123 & 0,019 & 68,4 & 10,8 & 79,2 \\
\hline 12 ou mais & 5,5 & 0,806 & 11,6 & 0,832 & 0,051 & 0,001 & 28,3 & 0,8 & 29,1 \\
\hline Total & 100 & 0,408 & 100 & 0,588 & 0,069 & 0,111 & 38,3 & 61,7 & 100,0 \\
\hline Educação Média & 4,0 & & 6,7 & & & & & & \\
\hline
\end{tabular}

Podemos resumir os resultados desta seção, que buscou mostrar as características das mulheres e a evolução da TPFT no período 1982-2002, bem como desagregar a evolução da TPFT entre efeito tamanho e efeito incidência. Fica claro o papel que a alteração do nível educacional teve no processo de aumento da TPFT feminina, já que quando o mesmo é levado em consideração, o efeito tamanho passa a responder por cerca de $38 \%$ do aumento da TPFT. Da mesma forma, o fato de que em todos os demais recortes da amostra o efeito tamanho foi muito pequeno reforça o papel da educação neste processo.

Por outro lado, somando os efeitos para cada categoria, podemos identificar quais mulheres mais contribuíram para o crescimento da TPFT. De acordo com os recortes da amostra, as maiores contribuições vieram das mulheres: brancas ou cônjuges ou com 8 a 11 anos de estudo ou com 1 filho ou com 40 a 44 anos.

Entretanto, não necessariamente o grupo das mulheres com todas estas características foi o que experimentou maior crescimento da TPFT. A precisão dos resultados desta análise seria prejudicada, já que os subgrupos cor-posição familiar-educação-filhos-idade ficariam muito reduzidos. Desta forma, na seção a seguir, iremos adotar uma metodologia que procurará solucionar este problema e que serve de teste de robustez para os resultados aqui obtidos.

\section{DECOMPOSIÇÃo POR CÉlULAS}

A verificação de que grupo de mulheres experimentou maior crescimento da participação feita anteriormente não considerou simultaneamente todas as características. Entretanto, construir células com todas as variáveis gerariam resultados não confiáveis, já que o tamanho das células ficaria muito pequeno (menos de trinta observações na maioria delas). Deste modo, nesta seção selecionamos apenas algumas das características abordadas na seção anterior. Optamos por trabalhar com 48 células, construídas de acordo com: anos de estudo ( 0 a 3, 4 a 7, 8 a 11 e 12 ou mais anos de estudo); posição na família (chefe, cônjuge e outra posição); idade (entre 25 e 44 anos e entre 45 e 64 anos); cor (brancas ${ }^{6}$ e não brancas). Esta escolha das subdivisões de cada variável, que não deixa

6 A categoria "branca" incluiu também as mulheres da categoria "amarela". 
de ser $a d-h o c$, foi feita no sentido de permitir que a maior parte das células tivesse, no mínimo, 50 mulheres. ${ }^{7}$

A construção das células permite identificar os grupos que apresentaram o maior crescimento da TPFT, bem como a importância relativa das mudanças de tamanho dos mesmos entre 1982 e 2002. A metodologia utilizada para o cálculo da decomposição da participação em efeito tamanho e efeito incidência com o uso de células baseou-se em Fernandes e Portela-Souza (2003). Entretanto, adaptamos a notação utilizada pelos autores aos termos já utilizados neste trabalho. Deste modo, podemos definir a variação na TPFT entre 1982 e 2002 como:

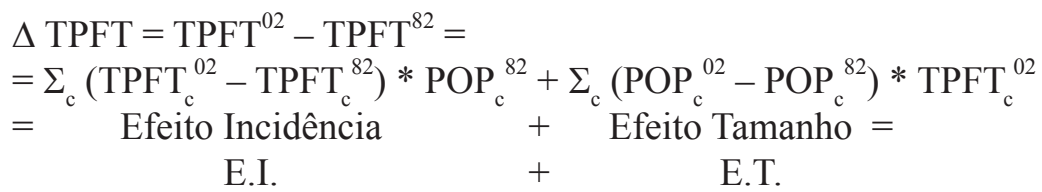

onde c identifica as 48 células mencionadas. Portanto, a equação (2) é simplesmente uma generalização da equação (1) para uma decomposição para c células, em vez de para cada um dos i grupos analisados isoladamente na seção anterior.

Antes de apresentarmos a decomposição representada na equação (2) seria interessante discutirmos a TPFT e o tamanho relativo das células, buscando identificar aquelas em que houve maior (ou menor) variação da TPFT, bem como as que tiveram as maiores variações de tamanho entre 1982 e 2002.

Tabela 8 - Células com as maiores e menores variações de tamanho e TPFT

\begin{tabular}{lcccccc}
\hline Célula & $\mathrm{POP}_{\mathrm{c}}^{82}$ & $\mathrm{POP}_{\mathrm{c}}{ }^{02}$ & $\mathrm{TPFT}_{\mathrm{c}}{ }^{82}$ & $\mathrm{TPFT}_{\mathrm{c}}{ }^{02}$ & $\Delta \mathrm{POP}_{\mathrm{c}}$ & $\Delta \mathrm{TPFT}_{\mathrm{c}}$ \\
\hline$(0,1,0,0)$ & 12,1 & 5,3 & 0,320 & 0,472 & $-6,80$ & 0,152 \\
$(0,1,0,1)$ & 11,3 & 3,2 & 0,311 & 0,476 & $-8,05$ & 0,165 \\
$(0,2,0,0)$ & 1,5 & 0,6 & 0,585 & 0,416 & $-0,85$ & $-0,169$ \\
$(0,2,0,1)$ & 1,3 & 0,4 & 0,511 & 0,388 & $-0,83$ & $-0,124$ \\
$(1,1,0,1)$ & 11,2 & 7,3 & 0,315 & 0,533 & $-3,86$ & $\mathbf{0 , 2 1 8}$ \\
$(2,1,0,1)$ & 3,0 & 4,2 & 0,337 & 0,580 & 1,19 & $\mathbf{0 , 2 4 3}$ \\
$(3,0,0,0)$ & 0,3 & 1,7 & 0,941 & 0,879 & 1,45 & $-0,062$ \\
$(3,0,0,1)$ & 0,8 & 3,1 & 0,933 & 0,895 & 2,26 & $-0,038$ \\
$(3,1,0,0)$ & 1,1 & 3,8 & 0,654 & 0,748 & $\mathbf{2 , 6 7}$ & 0,093 \\
$(3,1,0,1)$ & 5,7 & 9,0 & 0,617 & 0,750 & 3,36 & 0,133 \\
$(3,2,1,0)$ & 0,0 & 0,1 & 0,815 & 0,661 & 0,11 & $-0,154$ \\
\hline
\end{tabular}

Legenda: (anos de estudo, posição na família, idade e raça)

\begin{tabular}{lll} 
Anos de estudo: & $\begin{array}{l}0-0 \text { a } 3 \text { anos de estudo } \\
1-4 \text { a } 7 \text { anos de estudo }\end{array}$ & $\begin{array}{l}2-8 \text { a } 11 \text { anos de estudo } \\
3-12 \text { ou mais anos de estudo }\end{array}$ \\
\hline Posição na família & $\begin{array}{l}0-\text { chefe } \\
1-\text { cônjuge }\end{array}$ & $2-$ outras \\
\hline Idade & $0-25$ a 44 anos & $1-45$ a 64 anos \\
\hline Cor & $0-$ Não branca & $1-$ Branca
\end{tabular}

7 Mesmo assim, para o ano de 1982 duas células apresentaram menos de 50 observações: a das mulheres com 4 a 7 anos de estudo, em outra posição familiar, com idade de 45 a 64 anos e não brancas (34 mulheres) e a célula das mulheres com 8 a 11 anos de estudo, em outra posição familiar, com idade de 45 a 64 anos e não brancas (42 mulheres). 
A Tabela 8 apresenta as células que tiveram as maiores e menores variações de tamanho e de TPFT entre 1982 e 2002. A primeira coluna da tabela representa as células. Assim, a célula $(0,1,0,0)$ representa o grupo das mulheres com as seguintes características: 0 a 3 anos de estudo, cônjuges, com 25 a 44 anos e não brancas, conforme a legenda da Tabela 8.

As mulheres com o perfil de " 0 a 3 anos de estudo-cônjuges- 25 a 44 anos", independente da cor da pele (células $(0,1,0,0)$ e $(0,1,0,1)$ ), perfaziam o grupo mais numeroso em 1982 e o que percebeu a maior redução de tamanho até 2002. Por outro lado, o grupo das mulheres "com 12 ou mais anos de estudo-cônjuges-25-44 anos", independente da raça (células $(3,1,0,0)$ e $(3,1,0,1)$ ), foi o que mais cresceu entre 1982 e 2002, sendo que as brancas com este perfil perfaziam 9\% das mulheres em 2002. Estes dois movimentos indicam a importância da mudança do perfil educacional das mulheres na alteração do tamanho dos grupos no período.

Com relação à TPFT, as mulheres com " 0 a 3 anos de estudo-outra posição na família-25-44 anos" (células $(0,2,0,0)$ e $(0,2,0,1))$ e as com 12 ou mais anos de estudo-outra posição-45-64 anosnão brancas" (célula $(3,2,1,0)$ ) experimentaram as maiores quedas de TPFT. Por sua vez, as mulheres com " 8 a 11 anos de estudo-cônjuges-25-44 anos-brancas" tiveram o maior crescimento da TPFT, seguidas pelas mulheres com o mesmo perfil, mas com 4 a 7 anos de estudo.

Adicionalmente, cabe destacar que as células com as maiores TPFT em 1982 e 2002 eram as que continham mulheres com 12 ou mais anos de estudo, chefes de família e com idade entre 25 e 44 anos (células $(3,0,0,0)$ e $(3,0,0,1))$. Note que também para as mulheres com este perfil a cor da pele não possuía praticamente qualquer influência sobre a TPFT.

Apesar de a Tabela 8 permitir identificarmos as maiores variações, para que possamos averiguar a importância dos efeitos incidência e tamanho devemos analisar as variações de todas as células. Deste modo, a Tabela 9 apresenta os resultados da decomposição referente à equação (2):

Tabela 9 - Efeito incidência e efeito tamanho - 1982-2002

\begin{tabular}{lcc}
\hline & Pontos Porcentuais & Porcentagem \\
\hline Efeito Incidência & 0,109 & $60,41 \%$ \\
Efeito Tamanho & 0,071 & $39,59 \%$ \\
Variação Total & 0,180 & $100 \%$ \\
\hline
\end{tabular}

Com a decomposição sendo realizada por células, os resultados são diferentes dos obtidos na seção 2. Vimos anteriormente que, exceto para a divisão por grupos de educação, o efeito tamanho respondia por pequena parcela da evolução da TPFT. Deste modo, a existência de um efeito tamanho significativo para a variável educação levantou a hipótese de que a mudança de composição educacional tivesse afetado as mulheres com diferentes características (além da educação), o que poderia ser captado na análise por células. De fato, a análise dos resultados da Tabela 9 permite dizer que dos 18 pontos porcentuais de aumento da TPFT, cerca de $40 \%$ corresponde ao efeito de mudanças no tamanho das células, resultado semelhante ao encontrado na Tabela 7. Deste modo, os resultados confirmam a importância da alteração do tamanho dos grupos educacionais na explicação da variação da TPFT feminina.

Um passo adicional na análise seria decompor os efeitos incidência e tamanho "para verificar a importância de cada grupo, dentro de cada característica individual." (Fernandes e Portela-Souza, 2003, p. 7). Assim, por exemplo, para a variável idade, podemos saber a importância relativa de cada grupo etário (k) na variação dos efeitos incidência e tamanho. A variável idade possui 2 grupos, ou 
seja, $\mathrm{k}=1$ para mulheres entre 25 e 44 anos e $\mathrm{k}=2$ para mulheres entre 45 e 64 anos. Neste caso, metade das 48 células possui mulheres do grupo k. Deste modo, denotando $c k$ com as células que possuem mulheres do grupo etário k, podemos escrever:

$$
\begin{aligned}
& \text { Efeito Incidência }=\Sigma_{\mathrm{k}} \Sigma_{\mathrm{ck}}\left(\mathrm{TPFT}_{\mathrm{ck}}{ }^{02}-\mathrm{TPFT}_{\mathrm{ck}}{ }^{82}\right) . \mathrm{POP}_{\mathrm{ck}}{ }^{82} \\
& \text { Efeito Tamanho }=\Sigma_{\mathrm{k}} \Sigma_{\mathrm{ck}}\left(\mathrm{POP}_{\mathrm{ck}}{ }^{02}-\mathrm{POP}_{\mathrm{ck}}{ }^{82}\right) . \mathrm{TPFT}_{\mathrm{ck}}{ }^{02}
\end{aligned}
$$

Ainda seguindo Fernandes e Portela-Souza (2003), podemos definir:

$$
\begin{aligned}
& \mathrm{POP}_{\mathrm{ck}}^{*}{ }_{\mathrm{ck}}^{82}=\mathrm{POP}_{\mathrm{ck}}{ }^{82} / \Sigma_{\mathrm{ck}} \mathrm{POP}_{\mathrm{ck}}{ }^{82}=\mathrm{POP}_{\mathrm{ck}}{ }^{82} / \mathrm{POP}_{\mathrm{k}}^{+82} \mathrm{e} \\
& \mathrm{TPFT}_{\mathrm{ck}}^{*}{ }^{02}=\mathrm{TPFT}_{\mathrm{ck}}{ }^{02} / \Sigma_{\mathrm{ck}} \mathrm{TPFT}_{\mathrm{ck}}{ }^{02}=\mathrm{TPFT}_{\mathrm{ck}}{ }^{02} / \mathrm{TPFT}_{\mathrm{k}}^{+02}
\end{aligned}
$$

Deste modo, podemos escrever os efeitos incidência e tamanho como:

$$
\begin{aligned}
& \text { Efeito Incidência }=\Sigma_{\mathrm{k}}\left[\Sigma_{\mathrm{ck}}\left(\mathrm{TPFT}_{\mathrm{ck}}{ }^{02}-\mathrm{TPFT}_{\mathrm{ck}}{ }^{82}\right) \cdot \mathrm{POP}_{\mathrm{ck}}{ }^{82}\right] \cdot \mathrm{POP}_{\mathrm{k}}^{+82} \\
& \text { Efeito Tamanho }=\Sigma_{\mathrm{k}}\left[\Sigma_{\mathrm{ck}}\left(\mathrm{POP}_{\mathrm{ck}}{ }^{02}-\mathrm{POP}_{\mathrm{ck}}{ }^{82}\right) . \mathrm{TPFT}_{\mathrm{ck}}^{*}{ }^{02}\right] . \mathrm{TPFT}_{\mathrm{k}}^{+02}
\end{aligned}
$$

Assim, o termo entre colchetes em (3a) indica, para as mulheres do grupo etário k, a média das mudanças na TPFT entre 1982 e 2002, enquanto que $\mathrm{POP}_{\mathrm{k}}^{+82}$ representa a proporção de mulheres do grupo etário k na população total de mulheres em 1982. Por sua vez, o termo entre colchetes em (3b) representa a média de variação do tamanho relativo das células que possuem mulheres do grupo etário $\mathrm{k}^{8}$

Aplicando o procedimento de decomposição baseado em (3a) e (3b), construímos a Tabela 10.

$\mathrm{Na}$ Tabela 10, a coluna 1 contém o peso de cada um dos grupos, dado pela soma da TPFT de todas as células com aquelas características (ou seja, $\mathrm{TPFT}_{\mathrm{k}}^{+02}$ ). ${ }^{9}$ Assim, quanto maior o valor, maior a participação do grupo. Por exemplo, para a variável educação, a maior parte das mulheres participantes tinha mais que 11 anos de estudo, seguidas pelas mulheres com 8 a 11 anos de escolaridade. Na coluna 2, aponta-se a alteração do tamanho de cada grupo entre 1982 e 2002. A coluna 3 resulta da multiplicação das colunas 1 e 2 e fornece a contribuição de cada grupo (em cada variável) para o efeito tamanho. A coluna 4 contém o peso de cada grupo, dado pela proporção de mulheres em cada categoria em 1982 (ou seja, POP $_{\mathrm{k}}^{+82}$ ). Na coluna 5 mostra-se a variação da TPFT dentro de cada grupo (em cada variável). Por fim, a coluna 6 resulta do produto das colunas 4 e 5 , fornecendo a contribuição de cada grupo para o efeito incidência.

8 Para mais detalhes sobre a metodologia empregada, ver Fernandes e Portela-Souza (2003).

9 Note que para cada variável a soma dos pesos em cada grupo é igual a 28,48. Deste modo, podemos afirmar que, em 2002, $18,89 \%(5,25 / 28,48)$ das mulheres que participavam tinham de 0 a 3 anos de estudo. 
Tabela 10 - Efeito incidência e efeito tamanho desagregado por variáveis

\begin{tabular}{|c|c|c|c|c|c|c|}
\hline & \multicolumn{3}{|c|}{ Efeito Tamanho } & \multicolumn{3}{|c|}{ Efeito Incidência } \\
\hline & Peso & Dentro & E. T. & Peso & Dentro & E. I. \\
\hline \multicolumn{7}{|c|}{ Anos de estudo } \\
\hline 0 a 3 & 5,250 & $-0,021$ & $-0,108$ & 0,511 & 0,091 & 0,047 \\
\hline 4 a 7 & 6,806 & 0,001 & 0,004 & 0,291 & 0,148 & 0,043 \\
\hline 8 a 11 & 7,574 & 0,005 & 0,041 & 0,071 & 0,144 & 0,010 \\
\hline \multirow[t]{2}{*}{12 ou mais } & 8,852 & 0,015 & 0,135 & 0,126 & 0,069 & 0,009 \\
\hline & & & 0,071 & & & 0,109 \\
\hline \multicolumn{7}{|c|}{ Posição familiar } \\
\hline Chefe & 10,905 & 0,007 & 0,079 & 0,145 & 0,001 & 0,000 \\
\hline Cônjuge & 8,296 & $-0,002$ & $-0,015$ & 0,731 & 0,156 & 0,114 \\
\hline \multirow[t]{2}{*}{ Outra } & 9,280 & 0,001 & 0,007 & 0,124 & $-0,043$ & $-0,005$ \\
\hline & & & 0,071 & & & 0,109 \\
\hline \multicolumn{7}{|l|}{ Idade } \\
\hline 25 a 44 & 16,398 & 0,002 & 0,039 & 0,662 & 0,118 & 0,078 \\
\hline \multirow[t]{2}{*}{45 a 64} & 12,084 & 0,003 & 0,032 & 0,338 & 0,090 & 0,030 \\
\hline & & & 0,071 & & & 0,109 \\
\hline \multicolumn{7}{|l|}{ Cor } \\
\hline Não branca & 14,563 & 0,004 & 0,053 & 0,390 & 0,077 & 0,030 \\
\hline \multirow[t]{2}{*}{ Branca } & 13,919 & 0,001 & 0,018 & 0,610 & 0,129 & 0,079 \\
\hline & & & 0,071 & & & 0,109 \\
\hline
\end{tabular}

De modo geral, os resultados da Tabela 10 confirmam os achados da seção anterior. Em particular, cabe destacar alguns dos resultados. No que tange ao efeito tamanho, a redução do grupo com até 3 anos de estudo contribuiu para a diminuição da TPFT no período. Por outro lado, o incremento do grupo com mais de 11 anos de estudo foi suficiente para compensar tal redução, visto que as mulheres mais educadas eram as maiores participantes. Em relação ao efeito incidência, os maiores acréscimos de TPFT se deram entre as mulheres com 4 a 7 anos de estudo, fazendo com que fossem responsáveis pela variação em 0,043 no efeito incidência, resultado muito próximo ao das mulheres com 0 a 3 anos de estudo (0,047). Portanto, as magnitudes dos efeitos incidência e tamanho, no caso dos grupos educacionais, estão em linha com os obtidos na seção 2.

$\mathrm{O}$ mesmo pode ser dito com relação à posição familiar: efeito tamanho positivo para as chefes e negativo para as cônjuges e efeito incidência positivo para as cônjuges, reforçando a conclusão de que cada vez mais mulheres cônjuges vêm entrando no mercado de trabalho, apesar da proporção de cônjuges ter se reduzido no período.

Em termos da desagregação por cor da pele, o aumento da participação das brancas foi maior que o das não brancas e, por serem maioria, contribuíram com a maior parte do efeito incidência. Por fim, a análise dos grupos etários aponta as mulheres com até 44 anos como responsáveis pela maior parte do efeito incidência. Isto está relacionado ao maior engajamento das gerações mais novas ao mercado de trabalho, que tem feito com que uma proporção maior de mulheres destas gerações se engaje no mercado de trabalho. 


\section{CONCLUSÃo}

Este trabalho procurou caracterizar o processo de inserção feminina no mercado de trabalho brasileiro. Diferente de estudos anteriores, que se preocuparam em identificar em quais setores da atividade econômica as mulheres passaram a atuar mais fortemente, procurou-se investigar as características socioeconômicas das mulheres participantes do mercado de trabalho. A principal conclusão a que se chegou é que o aumento do nível educacional no Brasil entre 1982 e 2002 contribui, de modo relevante, para o crescimento da TPFT feminina, dado que há uma relação positiva entre escolaridade e taxa de participação, identificada em outros trabalhos e também neste. ${ }^{10} \mathrm{~A}$ mudança no perfil educacional foi o aspecto que mais se refletiu na mudança da composição dos grupos socioeconômicos. Deste modo, a contínua melhoria do nível educacional da mulher brasileira deve continuar contribuindo para a inserção feminina no mercado de trabalho. Uma prova disto é que as mulheres com os maiores crescimentos de participação foram as brancas, cônjuges, com idade entre 25 e 44 anos e 4 a 11 anos de estudo.

Outro aspecto importante a ser ressaltado é que embora em magnitudes diferentes, mulheres com as mais diferentes características aumentaram sua participação. Vale destacar o caso das mulheres cônjuges, que apresentaram o maior crescimento da TPFT no período. Entretanto, a entrada das cônjuges no mercado de trabalho poderia ser mais forte ainda caso houvesse políticas públicas com o objetivo de aumentar o custo de oportunidade do "não trabalho". Apesar de o avanço educacional ser uma política que reduz o custo de oportunidade do trabalho remunerado no mercado, seus efeitos são geracionais. Assim, é necessária uma intervenção de curto prazo para a solução deste problema. Desta forma, uma política agressiva de acesso a creches diminuiria as dificuldades para que as mulheres pudessem contribuir cada vez mais com a geração de renda familiar, principalmente as com baixo nível educacional.

\section{REFERÊNCIAS BIBLIOGRÁFICAS}

Blau, F.; Ferber, M.; Winkler, A. The economics of women, men, and work. 4 ed. Upper Saddle River: Prentice Hall, 2002.

Bruschini, C.; Lombardi, M. R. O trabalho da mulher brasileira nos primeiros anos da década de noventa. In: Encontro Nacional de Estudos Populacionais, 10. Anais. Belo Horizonte: ABEP, 1996, v.1, pp. 483-516.

Fernandes, R.; Portela Souza, A. A redução do trabalho infantil e o aumento da frequência a escola: uma análise de decomposição para o Brasil nos anos 90. In: Encontro Nacional da Associação Brasileira de Estudos do Trabalho, 8. Anais em Cd-Rom. São Paulo: ABET, 2003.

Guerra, V. S. Principais mudanças introduzidas nos conceitos e definições da PNAD para as pesquisas de 1992 em diante. 1992. Mimeografado.

Killingsworth, M. R.; Heckman, J. J. Female labor supply: a survey. In: Ashenfelter, O.; Layard, R. (eds.), Handbook of labor economics. 1986, cap. 2, p. 102-204.

Scorzafave, L.; Menezes-Filho, N. Participação feminina no mercado de trabalho brasileiro: evolução e determinantes. Pesquisa e Planejamento Econômico, v. 31, n. 3, p. 441-478, 2001.

Wajnman, S.; Queiroz, B.; Liberato, V. O crescimento da atividade feminina nos anos noventa no Brasil. In: Encontro Nacional de Estudos Populacionais, 11. Anais. Belo Horizonte: ABEP, v. 2, p. 2429-2454, 1998.

10 Ver, por exemplo, Scorzafave e Menezes-Filho (2001). Para outros países, ver Blau, Ferber e Winkler (2002). 